\title{
Relajación muscular y depresión en pacientes con esclerosis múltiple: estudio de caso múltiple
}

\author{
Muscular relaxation and depression among patients diagnosed \\ with multiple sclerosis: a multiple-case study \\ *Juan Luis Soto, *Carlos Armando Soto, *José Guadalupe Pérez, \\ *José Aldo Hernández, **Gabriel Gastélum
}

Soto, J.L., Soto, C.A., Pérez, J.G., Hernández, J.A., \& Gastélum, G. (2018). Relajación muscular y depresión en pacientes con esclerosis múltiple: estudio de caso múltiple. Revista Ciencias de la Actividad Física UCM, N 19(1) enero-junio, 1-7. DOI: http://doi.org/10.29035/rcaf.19.1.4

\section{RESUMEN}

El objetivo de esta investigación fue medir el efecto del Entrenamiento en Relajamiento Muscular Progresivo de Edmund Jacobson en los niveles de Depresión de tres pacientes con Esclerosis Múltiple (EM). Participaron inicialmente y de forma voluntaria 8 sujetos, todos del género femenino, de clase media, quienes no contaban con ningún tipo prestaciones en salud. De dichos casos valorados, se eligieron a tres pacientes con diferentes niveles de depresión: primer caso (sin depresión), segundo caso (depresión leve) y tercer caso (depresión moderada). Ya que sólo se encontraron estos niveles de depresión en el grupo valorado, es decir no se encontró depresión grave, lo anterior de acuerdo con los resultados arrojados por el Inventario de Depresión de Beck. El trabajo se basa en un diseño de estudio de caso múltiple, donde cada caso debe ser tratado en forma individual para luego sacar conclusiones del cruce de dichos casos. Con resultados alentadores, ya que en las tres situaciones hubo mejoras, demostrando que dicho entrenamiento de relajación muscular tiene efectos positivos sobre los niveles de ansiedad de este tipo de pacientes. Además, en cuanto al análisis introspectivo de cada paciente reportaron mejoras no sólo en depresión, sino, en autoestima, comunicación, socialización, aprendizaje y aceptación de la enfermedad.

\section{PALABRAS CLAVE}

Esclerosis múltiple, Depresión, Efectividad, Entrenamiento personalizado, Relajación muscular.

\begin{abstract}
The purpose of this research was to measure what the effect of a Progressive Muscular Relaxation Training of Edmund Jacobson (Year) would have on the different depression levels of 3 multiple sclerosis (MS) patients. Participants included 8 middle-class females, who did not have any type of health care. From those 8 participants, only 3 were selected that showed a different level of depression: first participant (No depression), second participant (low depression), and third participant (moderate depression). Since only these types of different levels of depression were found among the participants, severe depression was not found, though according to Beck's Depression Questionnaire (Year). This is a multiple-case study type of research, where each participant was treated individually, to better understand in an in depth manner the connection of these cases. We can say that our results are promising, since all three participants showed improvements, indicating a positive effect with the Progressive Muscular Relaxation Training over anxiety levels of participants. As well, according to an introspective analysis of each participant were shown improvements not only in depression levels but in self-esteem, socialization, communication skills, learning and adaptability to their disease.
\end{abstract}

\section{Key words}

Multiple sclerosis, Depression, Effectiveness, Personal training, Muscular relaxation.

\footnotetext{
* Facultad de Educación Física y Deporte, Universidad Autónoma de Sinaloa, México.

**Facultad de Ciencias de la Cultura Física, Universidad Autónoma de Chihuahua, México.
} 


\section{INTRODUCCIÓN}

La Esclerosis Múltiple a nivel mundial es la principal enfermedad desmielinizante del Sistema Nervioso Central, que afecta a individuos en su etapa más productiva de su vida y resulta de especial interés por su dificultad para ser diagnosticada, así como para conocer su probable etiología (Escobar et al. 2009).

Esta enfermedad también se caracteriza por ser crónica autoinmune, con inflamación focal, pérdida axonal y gliosis, afectando predominantemente a adultos jóvenes entre los 20 y 40 años (Grajales-González, Munive-Báez y de la Teja-Ángeles, 2013). Aunque esta tendencia está cambiando, ya que de acuerdo con Regalado y Fajardo (2014), en España se están descubriendo numerosos casos en la etapa adolescente.

La prevalencia de dicho padecimiento asciende a más de dos millones de personas en el mundo, su distribución atiende más a la genética que a la latitud geográfica. Además, afecta primordialmente a mujeres en una proporción de dos a uno, existiendo un alto riesgo de discapacidad neurológica importante (Noseworth, et al., 2000. Citado en Gutiérrez-Álvarez, 2006), así como diversos factores biopsicosociales: estrés psicológico, apoyo social percibido, bienestar psicológico, estrategias de afrontamiento) pueden tener un impacto clínico (López, Aliris, Olascoaga y Sistiaga, 2017).

Para Arango-Lasprilla, DeLuca y Chiaravalloti (2007), la atención oportuna y el conocimiento del perfil neurológico de las personas con Esclerosis Múltiple es de gran relevancia, tanto para el diagnóstico como para su posterior rehabilitación. Por su parte Miserda (2002), comenta que esta debe de ser considerada como una enfermedad de salud pública ya que el número de pacientes aumenta alarmantemente. A demás los avances de la medicina no han podido lograr una intervención más integral, desafortunadamente no existe cura para esta enfermedad.

Aunado a todo esto, los pacientes frecuentemente tienen dificultades para acceder a los tratamientos neurológicos, debido a su alto costo, de los más usados a nivel mundial se encuentran los Interferones, los cuales reducen un $30 \%$ los brotes en las formas remitente recurrente de la enfermedad (Gutiérrez-Álvarez, 2006).

En México, el estudio de esta enfermedad es casi nulo, los intentos por hacer una descripción de las características clínicas y demográficas son pocos, entre estos se encuentra el trabajo que realizaron Bertado-Cortés, Villamil-Osorio, Carrera-Pineda, Martínez-Cortés y Guerrero-Cantera (2016), en el Hospital de Especialidades del Centro Médico Nacional Siglo XXI.

La Fundación UNAM, estima que existen más de 20 mil casos de Esclerosis Múltiple, es decir, entre 18 y 15 incidencias por cada 100 mil habitantes. En el estado de Sinaloa, la Secretaría de Salud, no cuenta con datos sociodemográficos con respecto a esta enfermedad. Las únicas instituciones de salud en Sinaloa que ofrecen atención neurológica para pacientes con esta enfermedad son el ISSSTE (Instituto de Seguridad y Servicios Sociales de los Trabajadores del Estado) y IMSS (Instituto Mexicano del Seguro Social).

Los pacientes con EM manifiestan dos tipos de síntomas, los físicos y los psicológico-cognitivos en las diferentes etapas de la enfermedad. En relación a los primeros encontramos: cansancio extremo, entumecimiento de alguna parte del cuerpo, temblor en las manos, problemas de vista, pérdida de la fuerza muscular, entre los más conocidos. Pero el síntoma que Pérez-Belda (2012) considera más incapacitante, es la pérdida de la coordinación motriz. $\mathrm{Y}$ para una persona altamente productiva, resulta devastador saber que dejarán de hacer actividades que son fundamentales para su vida. Particularmente las mujeres experimentan una gran incertidumbre y angustia por que viven esperando el siguiente brote y con él, las cosa que dejarán cuando esto ocurra. Y aparece el "fantasma de la silla de ruedas", especialmente en mujeres independientes. 
Por otro lado, se encuentran los síntomas psicológicos, que se caracterizan por alteraciones en: el procesamiento de la información (Arango, et al. 2007); la memoria y la atención (Nieto, Sánchez, Barroso, Olivares y Hernández, 2008), la depresión y la autoestima, que son más frecuentes en las mujeres respecto a los varones (Aguilar, Eduardo y Berganza, 1996).

Así mismo, la ansiedad y la depresión (ya antes mencionada) son trastornos psiquiátricos vinculados a la EM. Pero según Olivares et al. (2009), no están relacionados con el deterioro neuropsicológico y se presentan generalmente en las etapas tempranas de la enfermedad. En el caso de la depresión es uno de los síntomas psicológicos más comunes en esta enfermedad, la mayoría de los pacientes evaluados reportan puntuaciones altas en la escala de depresión, además puede aparecer en cualquier atapa del padecimiento (Lara y Kirchener, 2012).

La mayoría de los pacientes con EM tienen que ser atendidos por "cuidadores", que regularmente es un familiar. El saber que alguien se tiene que hacer cargo de su vida, desarrolla un sentimiento de culpa por todos los cambios que la familia experimenta (pareja, hijos y económicos). En el caso de las mujeres este sentimiento es más aterrador, ya que ellas socialmente hablando son quienes proporcionan este cuidado a los integrantes de su familia (Pérez-Belda, 2012 y Bravo-González y Álvarez-Roldán, 2017).

La situación se agrava por la falta evidente de servicios médicos integrales para la atención de este tipo de pacientes. Aun cuando se sabe que la atención psicológica y física debe ser prioritaria, acompañando por supuesto a la atención neurológica. Por ejemplo, para Rodríguez, García y Cruz (2005), los beneficios del uso de las técnicas psicofisiológicas para el control de los estados psicoemocionales tales como el estrés, depresión, autoestima, entre otros, pueden ser muchísimas y dentro de las técnicas más utilizadas se encuentran la respiración y el relajamiento muscular progresivo. Se puede decir que, potencialmente, las técnicas de relajación, como la yoga y entre- namiento autógeno, concretamente, pueden brindar beneficios en diferentes dimensiones de la enfermedad (vitalidad, fatiga, depresión), pudiendo mejorar su calidad de vida de estos pacientes (Molina-Rueda y Pérez, 2009).

En un estudio realizado por Soriano (2012) donde utilizó la técnica de relajamiento muscular progresivo de Edmund Jacobson, que proponemos en nuestro trabajo, se obtuvieron excelentes resultados para el control de los estados emocionales, con la que entrenaron a 39 pacientes con diagnóstico de ansiedad, obteniendo buenos resultados en la reducción de los niveles de la enfermedad. Este investigador considera las terapias de relajación psicofisiológicas como importantes y alternativas a las farmacológicas, y de gran beneficio para la rehabilitación de este tipo de pacientes.

La calidad de vida de los pacientes con EM es muy deprimente, especialmente en las etapas avanzadas de la enfermedad. Para Arbinaga (2003), uno de los síntomas psicológicos que más afectan esta condición, es la depresión. En su estudio midió este síntoma en 17 pacientes con diagnóstico de EM, el instrumento que utilizó, precisamente, fue el Inventario de Depresión de Beck, y encontró que el 58 \% de ellos presentaron algún nivel depresivo.

Por lo anterior expuesto hasta aquí, a manera de un breve planteamiento del problema de investigación, comentamos que la Depresión en pacientes con Esclerosis Múltiple ha sido muy poco estudiada, así como los tratamientos para controlarla y mejorar los síntomas adversos. Por lo que consideramos que el entrenamiento en Relajamiento Progresivo puede ayudar al paciente a controlar, en estos casos, los niveles de Depresión en ellos.

El objetivo de esta investigación fue medir el efecto del Entrenamiento en Relajamiento Progresivo de Edmund Jacobson en los niveles de Depresión de tres pacientes con Esclerosis Múltiple. 


\section{METODOLOGÍA}

Este trabajo contempla un diseño de investigación en base a estudio de caso múltiple, con enfoque cualitativo.

Los criterios de inclusión considerados fueron los siguientes: Pacientes con Esclerosis Múltiple, que no habían recibido algún tipo de terapia física o psicológica. Se excluyeron a aquellos que presentaban limitaciones físicas graves para su traslado a las sesiones de intervención.

Por lo que, participaron en este estudio tres sujetos voluntarios, del género femenino, 45 años de promedio, todas diagnosticadas con Esclerosis Múltiple de tipo Remitente-Recurrente, con tres niveles de depresión. Caso 1: Sin depresión. Caso 2: Depresión leve. Caso 3: Depresión moderada. Ya que el diseño de investigación por el que se optó fue el estudio de caso múltiple, es decir de corte cualitativo, y aunque se midió con una escala cuantitativa la misma que da resultados cualitativos en cuanto al nivel de depresión, como se describe a continuación.

Se utilizó para medir los estados de ansiedad de los pacientes, antes y después de la aplicación del programa, el Inventario de Depresión de Beck (BDI). Este instrumento, fue estandarizado por Jurado et al., (1998) en población mexicana $(\alpha=.87)$, que es un autoinforme de 21 ítems, con cuatro opciones de respuesta. Los puntajes de severidad de síntomas van de 0 a 63, donde 63 representa la severidad máxima. El punto de corte establecido por el autor (Jurado et al., 1998) para población mexicana fue de 10 puntos. Así mismo, se usó un Formato de Entrevista, es decir, de autorreporte, donde se recogió información sociodemográfica de los pacientes.

La intervención de los sujetos fue por medio del programa de Entrenamiento Relajamiento Muscular Progresivo, que es una técnica desarrollada por el médico Edmund Jacobson en 1920, cuyo objetivo es reducir los niveles de ansiedad, estrés y depresión; aprendiendo a relajar la tensión muscular. El ejercicio en relajación progresiva favorece una relajación profunda sin apenas esfuerzo, permitiendo establecer un control voluntario de la tensióndistensión que llega más allá del logro de la relajación en un momento dado. Es un método que favorece un estado corporal más energético pues favorece intensamente el reposo; permite reconocer la unión íntima entre tensión muscular y estado mental tenso, mostrando con claridad como liberar uno, implicando liberar el otro. En todo momento se respetó y consideró la Declaración de Helsinki de la Asociación Médica Mundial, para los pacientes que participaron en la intervención, estableciéndose la prioridad del bienestar de cada uno de ellos sobre los intereses de la investigación.

Las etapas del Procedimiento constaron de siete momentos específicos:

1. Difusión del proyecto, haciendo hincapié en la invitación a pacientes con Esclerosis Múltiple para que participen de manera voluntaria en el mismo. Para lo que se pegaron carteles en las Unidades Académicas de la Universidad Autónoma de Sinaloa, Zona Centro y en los Principales Hospitales de la ciudad de Culiacán, Sinaloa (4 meses).

2. Aplicación del formato de Entrevista y del Inventario de Depresión a todos los pacientes interesados, a quienes se les entregaba los instrumentos cuando llegaban a las instalaciones de la FEFYDE UAS, donde lo contestaban (2 meses).

3. Selección de los pacientes, a partir de la aplicación de los instrumentos, antes mencionados, de entre 8 posibles voluntarios, se eligieron a tres sujetos que cumplían con los criterios de inclusión, a quien se les solicitó leer y firmar el formato de Consentimiento Informado para Participantes de Investigación.

4. Intervención, esta constó de un total de 25 sesiones de una hora, con dos sesiones por semana durante tres meses (septiembrenoviembre), cada sesión se distribuía de la siguiente manera: durante los primeros 25 minutos se impartía un taller sobre temas relacionados con la calidad de vida de los 
pacientes con Esclerosis Múltiple; y los 35 minutos restantes se dedicaba al entrenamiento de Relajamiento Muscular Progresivo, dedicando los últimos minutos de esta etapa al entrenamiento neuro-lingüístico.

5. Segunda y última aplicación del Inventario de Depresión.

6. Se realizó un taller vivencial, donde se les pidió a cada uno de los pacientes que describieran los cambios personales que experimentaron después de la intervención.

7. Recolección y análisis de los datos.

\section{RESULTADOS}

Se da una breve explicación sobrelos puntajes que arroja el Inventario de Depresión de Beck, así como su interpretación, para efectos de que los resultados sean entendidos de mejor manera.

Este inventario, como ya se dijo, es un autoinforme de 21 ítems, con cuatro opciones de respuesta (de 0 a 3 ), cuya puntuación máxima es de 63. Con cuatro situaciones de depresión, que va desde ausencia total de ésta hasta depresión grave, como se describe a continuación. No depresión: 0-9 puntos, Depresión leve: 1018 puntos, Depresión moderada: $19-29$ puntos y Depresión grave: $>30$ puntos.

Como se puede apreciar en la figura 1, el "Caso 1" que inicialmente fue valorado Sin Depresión, pero muy cercano a la Depresión Leve (que inicia a partir de un puntaje de 10), después del programa bajó aún más su puntaje. Por su parte, el "Caso 2" con Depresión Leve al inicio del programa, pasó al rango Sin Depresión. El “Caso 3” también mejoró, pasando de Depresión Moderada a Leve. Es decir, de manera general, los sujetos de nuestro estudio se beneficiaron del programa de relajación en cuanto a su estado de depresión.

Haciendo un esfuerzo por comparar los tres casos, analizando los resultados que se muestran en la figura 1 , se observan mejores resultados en el "Caso 1" luego en el "Caso 2" y por último el "Caso 3". Lo que nos hace pensar en la posibilidad de que este tipo de entrenamiento de relajación, es efectivo, sobre todo, en estados de ansiedad bajos.

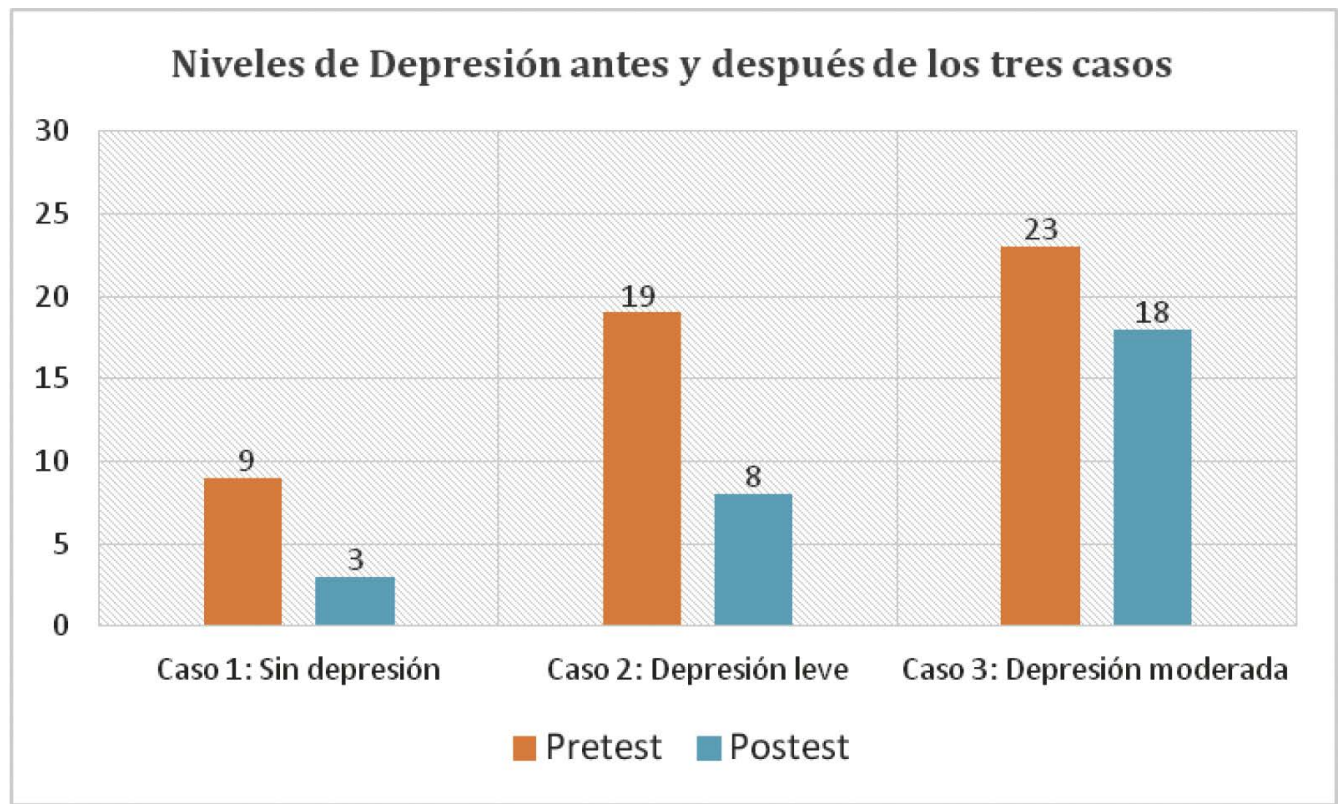

Figura 1. Puntajes obtenidos del Inventario de Depresión de Beck, antes y después del Programa de Entrenamiento Relajamiento Muscular Progresivo, de los tres casos de estudio. 


\section{DISCUSIÓN}

Es necesario reconocer que el diseño de investigación utilizado en este trabajo, estudio de caso, presenta fortalezas y debilidades. Por un lado, una de las bondades es que se trabaja con pocos sujetos, lo que permite una atención más personalizada en todos los sentidos. Por otro lado, los resultados obtenidos pierden capacidad de ser generalizados para esta población. Por lo que sugerimos se siga investigando sobre este tema.

Lo encontrado en nuestro trabajo coincide con Soriano (2012) quien reconoce a la técnica de relajamiento muscular progresivo de Edmund Jacobson, como una excelente herramienta para el control de estados emocionales. Además, este autor junto con Molina-Rueda y Pérez (2009), consideran que las terapias de relajación psicofisiológicas como importantes y alternativas a las farmacológicas, y de gran beneficio para la rehabilitación de este tipo de pacientes, pudiendo mejorar su calidad de vida.

En otro sentido, como se comentó en la sección de resultados, los tres pacientes que recibieron el programa se beneficiaron de él. Pero de acuerdo con los puntajes de la escala con que se midió, el primer caso fue el que logró bajar más su nivel de ansiedad, luego el segundo y posteriormente el tercero. Por lo que resaltamos su capacidad en la prevención de la depresión de estos pacientes. Es decir que recomendamos su utilización al poco tiempo del diagnóstico inicial de este padecimiento, pero sin descartar su uso en cualquier etapa de la enfermedad.

\section{CONCLUSIONES}

Queda demostrado el valor terapéutico de la técnica de relajamiento muscular progresivo de Edmund Jacobson, como una herramienta útil y alterna al tratamiento farmacológico. Y que la intervención es más efectiva, entre más cercana sea en relación al diagnóstico de este padecimiento o a los primeros indicios de depresión de los pacientes. Por lo que se reconoce su capacidad preventiva en la aparición y aumento de la depresión en esta población. Haciendo falta mayor investigación en este sentido, utilizando otros diseños, sobre todo donde se involucre una mayor cantidad de sujetos.

\section{REFERENCIAS BIBLIOGRÁFICAS}

Aguilar, G., Eduardo, H. y Berganza, C. (1996). Autoestima y depresión en adolescentes guatemaltecos. Revista Latinoamericana de Psicología, 28(2), 341-366.

Arango-Lasprilla, J., DeLuca, J. y Chiaravalloti, N. (2007). El perfil neuropsicológico en la esclerosis múltiple. Psicothema, 19(1), 1-6.

Arbinaga Ibarzábal, F. (2003). Aspectos emocionales y calidad de vida en pacientes con enfermedades desmielinizantes: el caso de la esclerosis múltiple. Anales de Psicología, 19(1).

Bertado-Cortés, B., Villamil-Osorio, L., Carrera-Pineda, R., Martínez-Cortés, C. y Guerrero-Cantera, J. (2016). Características clínicas y demográficas de los pacientes con esclerosis múltiple. Revista Médica del Instituto Mexicano del Seguro Social, 54(2), S186-S190.

Bravo-González, F., Álvarez-Roldán, A. (2017). Esclerosis múltiple, pérdida de funcionalidad y género. Gaceta Sanitaria. Recuperado: de https://www. sciencedirect.com/science/article/pii/ $\underline{\text { S0213911117302662 }}$

Escobar Darwin, V., Kawano Castillo, J., Castañeda Barba, C., Chereque Gutierrez, A., Tipismana Barbarán, M., Bernabé Ortiz, A. y Juárez Belaúnde, A. (2009). Prevalencia de Esclerosis Múltiple en Lima - Perú. Revista Médica Herediana, 20(3), 146-150.

Grajales-González, H., Munive-Báez, L. y de la Teja-Ángeles, E. (2013). Manifestaciones bucofaciales en pacientes con esclerosis múltiple. Acta Pediátrica de México, 34(5), 253-257. 
Gutiérrez-Álvarez, Á. (2006). Esclerosis múltiple: evidencias y controversias. Revista Ciencias de la Salud, 4(1), 52-58.

Jurado, S., Villegas, M. E., Méndez, L., Rodríguez, F., Loperena, V. y Varela, R. (1998). La estandarización del Inventario de Depresión de Beck para los residentes de la Ciudad de México. Salud Mental, 21, 26-31.

Lara Paredes, S. y Kirchner Nebot, T. (2012). Síntomas psicológicos en españoles enfermos de esclerosis múltiple: rol del género y relación con los años de diagnóstico. Revista Mexicana de Psicología, 29(2), 147-156.

López-Alava, S., Aliri, J., Olascoaga, J. y A. Sistiaga, A. (2017). Factores psicosociales y rendimiento cognitivo en esclerosis múltiple: diferencias de sexo. Revista de neurología, 65(5), 216-222.

Miserda P.R. (2002). Un nuevo paradigma propuesto para la esclerosis múltiple. Pharos, 9(1).

Molina-Rueda, F. y Pérez de la Cruz, S. (2009). Esclerosis Múltiple y Técnicas de Relajación. Revista Iberoamericana de Fisioterapia y Kinesiologia, 12(1), 28-34.

Nieto Barco, A., Sánchez López, M., Barroso Ribal, J., Olivares Pérez, T. y Hernández Pérez, M. (2008). Alteraciones cognitivas en etapas iniciales de la esclerosis múltiple y su relación con el estado de ánimo, variables demográficas y clínicas. Psicothema, 20(4), 583-588.

Olivares-Pérez, T., Nieto-Barco, A., BetancortMontesinos, M., Pérez- Martín, Y., Hernández-Pérez, M. y Barroso-Ribal, J. (2009). Ansiedad y depresión en la esclerosis múltiple remitente-recidivante: relación con las alteraciones neuropsicológicas y la percepción subjetiva de deterioro cognitivo en pacientes con discapacidad mínima/leve. Revista Chilena de Neuropsicología, 4(1), 44-51.
Pérez-Belda, M. (2012). Afrontando la Esclerosis Múltiple: Sentimientos y Temores. Portularia, XII (2), 117-125.

Regalado Cuenca, A. y Fajardo Caldera, M. (2014). Eficacia de un programa de psicología positiva en un adolescente con esclerosis múltiple. International Journal of Developmental and Educational Psychology, 1(1), 379-389.

Rodríguez Rodríguez, T., García Rodríguez, C. y Cruz Pérez, R. (2005). Técnicas de relajación y autocontrol emocional. MediSur, 3(3), 55-70.

Soriano González, J. (2012). Estudio cuasi-experimental sobre las terapias de relajación en pacientes con ansiedad. Enfermería Global, 11(2), 39-53.

\section{Dirección para correspondencia}

Gabriel Gastelum

Profesor de Educación Física. Dr. Facultad de Ciencias de la Cultura Física - Universidad Autónoma de Chihuahua, México.

Catedrático Núcleo Básico del Doctorado en Ciencias de la Cultura Física (UACH).

Cuerpo Académico 121: "Desarrollo personal, Actividad Física y Calidad de Vida".

Miembro del Sistema Nacional de Investigadores (SNI, Nível I).

Director Académico y de Planeación de la International Human Motricity Network (IHMN).

Contacto:

gastelum@uach.mx

Recibido: $23 / 02 / 2018$

Aceptado: 05/03/2018 